\title{
Влияние буферного слоя por-Si на структуру и морфологию эпитаксиальных гетероструктур $\ln _{x} \mathrm{Ga}_{1-x} \mathrm{~N} / \mathrm{Si}(111)$
}

\author{
(С) П.В. Середин ${ }^{1}$, Д.Л. Голощапов ${ }^{1}$, Д.С. Золотухин ${ }^{1}$, М.А. Кондрашин ${ }^{1}$, \\ А.С. Леньшин ${ }^{1}$, Ю.Ю. Худяков ${ }^{1}$, А.М. Мизеров ${ }^{2}$, И.Н. Арсентьев ${ }^{3}$, \\ A.Н. Бельтюков ${ }^{4}$, Harald Leiste ${ }^{5}$, Monika Rinke ${ }^{5}$ \\ ${ }^{1}$ Воронежский государственный университет, \\ 394006 Воронеж, Россия \\ ${ }^{2}$ Санкт-Петербургский национальный исследовательский Академический университет Российской академии наук, \\ 194021 Санкт-Петербург, Россия \\ ${ }^{3}$ Физико-технический институт им. А.Ф. Иоффе Российской академии наук, \\ 194021 Санкт-Петербург, Россия \\ ${ }^{4}$ Физико-технический институт Уральского отделения Российской академии наук, \\ 426000 Ижевск, Россия \\ ${ }^{5}$ Karlsruhe Nano Micro Facility, \\ 76344 Eggenstein-Leopoldshafen, Germany \\ E-mail: paul@phys.vsu.ru
}

(Получена 12 апреля 2018 г. Принята к печати 21 мая 2018 г.)

\begin{abstract}
С использованием метода молекулярно-пучковой эпитаксии с плазменной активацией азота на подложке монокристаллического кремния $c$ - $\mathrm{Si}(111)$ и подложке с нанопористым буферным подслоем (por-Si) нами были выращены интегрированные гетероструктуры с наноколончатой морфологией пленки $\operatorname{In}_{x} \mathrm{Ga}_{1-x} \mathrm{~N}$. С привлечением комплекса структурных и микроскопических методов анализа было показано, что рост наноколонок $\operatorname{In}_{x} \mathrm{Ga}_{1-x} \mathrm{~N}$ на нанопористом буферном слое имеет ряд преимуществ по сравнению с ростом на $c$-Si. Подложка por-Si задает преимущественную ориентацию роста наноколонок $\operatorname{In}_{x} \mathrm{Ga}_{1-x} \mathrm{~N}$ ближе к направлению ориентации $\mathrm{Si}(111)$, а также позволяет получить наноколонны $\operatorname{In}_{x} \mathrm{Ga}_{1-x} \mathrm{~N}$ с более высокой кристаллографической однородностью и унифицированным по всей поверхности латеральным размером наноколонн $\sim 40$ нм. Рост наноколонн $\operatorname{In}_{x} \mathrm{Ga}_{1-x} \mathrm{~N}$ на пористом слое por-Si приводит к снижению величины компонент деформации $\varepsilon_{x x}$ и $\varepsilon_{z z}$, а также плотности краевых и винтовых дислокаций по сравнению с величинами аналогичных коэффициентов для наноколонн $\operatorname{In}_{x} \mathrm{Ga}_{1-x} \mathrm{~N}$ выращенных на $c$-Si. Полученный на por-Si наноколончатый слой $\operatorname{In}_{x} \mathrm{Ga}_{1-x} \mathrm{~N}$ обладает более высокой концентрацией носителей заряда $(+20 \%)$ по сравнению со слоем, выращенный на $c$-Si, а также более высокой интенсивностью квантового выхода фотолюминесценции $(+25 \%)$.
\end{abstract}

DOI: $10.21883 /$ FTP.2018.13.46865.8888

\section{1. Введение}

Твердые растворы $\operatorname{In}_{x} \mathrm{Ga}_{1-x} \mathrm{~N}$ являются идеальными кандидатами для разнообразных оптоэлектронных применений. Благодаря тому, что ширина запрещенной зоны $\mathrm{In}_{x} \mathrm{Ga}_{1-x} \mathrm{~N}$ может изменяться в диапазоне энергий от 0.7 до 3.4 эВ, т. е. в зависимости от содержания индия может располагаться как в ИФ, так и в УФ диапазоне, эти твердые растворы активно используются как при конструировании свето- и лазерных диодов, так и солнечных элементов $[1,2]$

Хорошо известно, что основной подложкой для эпитаксиального роста $\mathrm{A}^{\mathrm{III}} \mathrm{N}$ соединений с гексагональной кристаллической решеткой является сапфир $c-\mathrm{Al}_{2} \mathrm{O}_{3}$. Однако использование монокристаллического кремния $(c-\mathrm{Si})$ в роли подложки и его комбинация с $\mathrm{A}^{\mathrm{III}} \mathrm{N}$ также весьма часто используется и имеет ряд преимуществ для промышленности, обусловленных уже тем фактом, что кремний - это основной материал современной электроники.

Однако эпитаксиальный рост слоев $\operatorname{In}_{x} \mathrm{Ga}_{1-x} \mathrm{~N}$, подходящих для оптоэлектронных устройств на подлож- ках $\mathrm{Si}$, оказался не простой задачей [2-4]. Внушительная разница между постоянными кристаллической решетки и коэффициентами теплового расширения у твердых растворов $\mathrm{A}^{\mathrm{III}} \mathrm{N}$ и подложки $\mathrm{Si}$ весьма серьезно затрудняет рост пленки хорошего качества. Кроме того, при росте тройного твердого раствора $\operatorname{In}_{x} \mathrm{Ga}_{1-x} \mathrm{~N}$ часто происходит либо разделение фаз, либо имеет место небольшая флуктуация состава, что приводит к появлению структурных дефектов, действующих как центры безызлучательной рекомбинации. Еще одним фактором, влияющим на качество пленок и соответственно коммерческую привлекательность финальных устройств на основе $\operatorname{In}_{x} \mathrm{Ga}_{1-x} \mathrm{~N}$, является тот факт, что технологам все еще не удалось преодолеть текущие трудности с созданием гетероструктур, в основе которых находятся низкодефектные монокристаллические твердые растворы $\operatorname{In}_{x} \mathrm{Ga}_{1-x} \mathrm{~N}$ с высоким содержанием индия в составе и низкой плотностью дислокаций.

Эти трудности все еще преодолеваются исследователями, однако разработка новых подходов к эпитаксиальному росту нитридов на Si с применением молекулярно- 
пучковой эпитаксии (МПЭ) уже позволила повысить качество пленки, получаемой на подложке кристаллического $\mathrm{Si}$ до уровня активных применений в оптоэлектронике [5].

Одним из перспективных подходов к интеграции $\mathrm{In}_{x} \mathrm{Ga}_{1-x} \mathrm{~N}$ и $\mathrm{Si}$, используемых в рамках МПЭ является создание наноколончатой структуры на поверхности подложки [6,7]. Выращенные в виде нанопроводов/наностобиков твердые растворы $\operatorname{In}_{x} \mathrm{Ga}_{1-x} \mathrm{~N}$ являются уникальной платформой для дальнейшего роста гетероструктур. Хорошо известно, что именно в этой геометрии может быть значительно снижена энергия упругих напряжений, вызванная несоответствием параметров кристалличеких решеток в гетеропаре $\operatorname{In}_{x} \mathrm{Ga}_{1-x} \mathrm{~N} / \mathrm{Si}[8,9]$.

Снижение упругой энергии, возникающей из-за несоответствия параметров решетки, с помощью создания слоя наностолбиков и их последующей коалесценции, может играть важную роль в последующем росте на них низкодефектной монокристаллической пленки $\operatorname{In}_{x} \mathrm{Ga}_{1-x} \mathrm{~N}$.

Еще одним весьма перспективным подходом для роста слоя $\mathrm{A}^{\mathrm{III}} \mathrm{B}^{\mathrm{V}}$ на кремнии является использование буферного слоя нанопористого Si. В наших предыдущих работах было показано, что рост на нанопрофилированной поверхности кремния с существенно развитым рельефом (протопористый кремний) способствует интеграции $\mathrm{A}^{\mathrm{III}} \mathrm{B}^{\mathrm{V}}$ материалов с Si. B экспериментах по росту GaAs на por-Si были отмечены значительно меньшие остаточные напряжения в монокристаллической пленке и ее рост без использования переходного слоя в виде наноколончатой структуры [10-12].

Мы полагаем, что такой подход по созданию „податливых“ подложек, в основе которых находятся нанопористый буферный слой, сформированный непосредственно на $\mathrm{Si}$, и выращенная на нем наноколончатая структура, является весьма перспективным подходом к интеграции $\mathrm{A}^{\mathrm{III}} \mathrm{N}$ материалов с кремнием.

Обзор литературы по поставленной проблеме показал отсутствие аналогичных работ. Поэтому цель нашей работы состояла в исследовании влияния буферного слоя por-Si на особенности гетероэпитаксиального роста и физические свойства гетероструктур на основе соединений $\operatorname{In}_{x} \mathrm{Ga}_{1-x} \mathrm{~N}$ с наноколончатой морфологией пленки.

\section{2. Материалы и методы}

Для исследования влияния буферного слоя por-Si на особенности гетероэпитаксиального роста и физические свойства гетероструктур на основе соединений (In, Ga)N/Si c наноколончатой морфологией пленки в едином эпитаксиальном процессе была выращена серия тестовых образцов. Были получены два типа образцов гетероструктур: (In, Ga)N/Si(111) (образец $\left.a \_c r y s t\right)$ и (In,Ga)N/por-Si/Si(111) (образец a_por).
Буферный слой пористого кремния в структуре a_por мы получали методом электрохимического травления монокристаллических пластин кремния ориентации (111) в спиртовом растворе плавиковой кислоты по стандартной методике [13-15]. Толщина пористого слоя составляла $\sim 30$ нм. Средний размер пор 5-10 нм.

Слои $\operatorname{~}_{x} \mathrm{Ga}_{1-x} \mathrm{~N}$ с наноколончатой морфологией пленки выращивались методом молекулярно-пучковой эпитаксии с плазменной активацией азота (МПЭ ПА) на установке промышленного типа Veeco Gen 200 на подложках, прошедших предварительную химическую подготовку по методу Шираки. Удаление защитного слоя оксида кремния с подложек осуществлялось во время их отжига в ростовой камере при температуре подложки $T_{S}=850^{\circ} \mathrm{C}$ в течение 30 мин. Слой $\operatorname{In}_{x} \mathrm{Ga}_{1-x} \mathrm{~N}$ толщиной $\sim 150$ нм выращивался при постоянных значениях температуры подложки $T_{S}=400^{\circ} \mathrm{C}$ в единичных стехиометрических условиях роста при равных соотношениях потоков металлов третьей группы $\left(F_{\mathrm{III}}=F_{\mathrm{Ga}}+F_{\mathrm{In}}\right)$ и активированного азота $F_{\mathrm{III}} \sim F_{\mathrm{N}} \sim 0.06$ мкм/ч, где поток индия составлял $F_{\text {In }} \sim 0.02$ мл/с, поток галлия $F_{\mathrm{Ga}} \sim 0.04$ мл/с.

Микроскопические исследования качества гетероинтерфейсов были выполнены на электронном микроскопе Libra 120 Carl Zeiss. Изучение морфологии поверхности проводили с использованием сканирующего зондового микроскопа Femtoscan-001 NT MDT в режиме атомносиловой микроскопии.

Рентгенофазовый анализ полученных образцов проводили методом рентгеновской дифракции, с использованием дифрактометра ДРОН 4-07 с кобальтовой трубкой $\mathrm{Co} K_{\alpha}=1.790 \AA$.

Структурное качество образцов, определение параметров решеток твердых растворов (кривые качания), а также анализ ориентации полученных наноколонн с использованием полюсных фигур проводили методом рентгеновской дифракции на дифрактометре Seifert 3003 HR с четырехкружным гониометром и монохроматизированным излучением меди с длиной волны $\mathrm{Cu} K_{\alpha 1}=1.5405 \AA$.

Концентрации элементов в твердом растворе были уточнены методом рентгеновского микроанализа с использованием приставки к электронному микроскопу.

Концентрация носителей и тип проводимости определялись с помощью эффекта Холла методом Ван дер Пау при комнатной температуре с использованием установки Ecopia-3000 (Корея). Планарные серебряные контакты толщиной 30 нм были нанесены на пленки методом магнетронного напыления.

\section{3. Экспериментальные результаты}

\section{1. Электрофизические измерения}

Измерения типа проводимости, концентраций, а также подвижности носителей заряда методом Ван дер Пау 

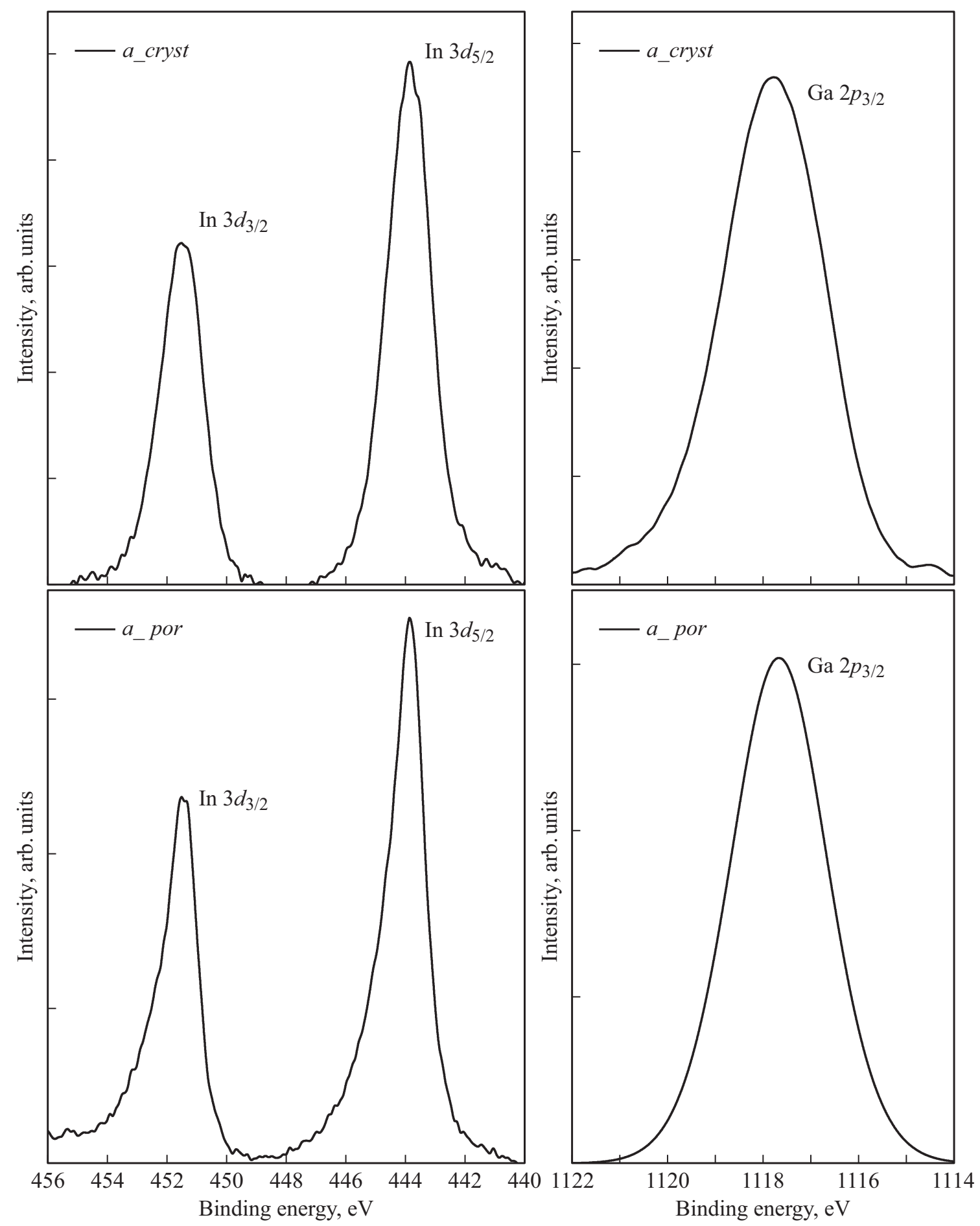

Рис. 1. Результаты XPS-спектроскопии.

при комнатной температуре показали, что наноколончатая пленка $\operatorname{In}_{x} \mathrm{Ga}_{1-x} \mathrm{~N}$ для образцов обоих типов имеет $p$-тип проводимости. При этом величина холловской концентрации примеси находится на уровне (3.0-3.64) $\cdot 10^{19} \mathrm{~cm}^{-3}$, а подвижность носителей заряда $\sim 104 \mathrm{~cm}^{2} / \mathrm{B} \cdot \mathrm{c}$ ) (табл. 1). Отметим, что если подвижность носителей для образцов находится на одном уровне, то концентрация носителей заряда в слое, вы- ращенном на пористом кремнии $p o r-\mathrm{Si}$, на $\sim 20 \%$ выше, чем для слоя на $c$-Si.

\section{2. Фазовый анализ}

Используя приставку для рентгеновского микроанализа к электронному микроскопу, мы определили концентрации элементов, входящих в состав наноколончатой 
Таблица 1. Описание образцов и результаты электрофизических методов измерений

\begin{tabular}{c|c|c|c}
\hline Образец & $\begin{array}{c}\text { Состав, } \\
x\end{array}$ & $\begin{array}{c}\text { Концентрация } \\
\text { носителей заряда, } \mathrm{cm}^{-3}\end{array}$ & $\begin{array}{c}\text { Подвижность, } \\
\mathrm{cm}^{2} / \mathrm{B} \cdot \mathrm{c}\end{array}$ \\
\hline $\begin{array}{c}\text { a_cryst } \\
\text { a_por }\end{array}$ & 0.32 & $3.00 \cdot 10^{19}$ & 104.7 \\
& 0.34 & $3.64 \cdot 10^{19}$ & 103.4
\end{tabular}

структуры. Для анализа использовалось ускоряющее напряжение электронов 10 кВ и исследовались участки образца порядка $750 \times 750$ мкм. Эффективная глубина микроанализа составила $\sim 0.25$ мкм. Эксперимент показал, что концентрация атомов индия в твердом растворе находится на уровне $31-33 \%$ для обоих образцов. С применением метода рентгеновской фотоэлектронной спектроскопии (XPS), которая позволяет исследовать химический состав поверхности, мы уточнили концентрации атомов в твердом растворе $\operatorname{In}_{x} \mathrm{Ga}_{1-x} \mathrm{~N}$. XPS спектры Ga2 $p_{3}$ и In $3 d_{5}$ приведены на рис. 1 . Как видно из экспериментальных данных, максимум $\mathrm{Ga} 2 p_{3}$ расположен около 1117.0 эВ, в то время как In $3 d_{5}$ дублет около 444.2 и 452 эВ соответственно.

Как показано в работе [16], содержание индия в пленке может быть рассчитано на основе следующего соотношения:

$$
X_{\mathrm{In}}=\frac{I_{\mathrm{In} 3 d_{5}} / F_{\operatorname{In} 3 D_{5}}}{I_{\operatorname{In} 3 d_{5}} / F_{\operatorname{In} 3 D_{5}}+I_{\mathrm{Ga} 2 p_{3}} / F_{\mathrm{Ga} 2 P_{3}}},
$$

где $I$ - интегральная интенсивность фотоэлектронных максимумов соответствующих линий в спектре, $F-$ фактор чувствительности $\left(F_{\mathrm{Ga} 2 p_{3}}=2.75\right.$ и $\left.F_{\mathrm{In} 3 d_{5}}=4.53\right)$. Определенные на основе соотношения (1) значения концентрации атомов In составили $x_{a \_ \text {cryst }}=0.32$ и $x_{a \_p o r}=0.34$ для образцов $a \_c r y s t$ и $a \_p o r$ соответственно (см. табл. 1), что весьма хорошо согласуется с данными микроанализа.

Следует отметить, что исходя из анализа XPS спектров In $3 d_{5}$, поверхность наностолбиков $\operatorname{In}_{x} \mathrm{Ga}_{1-x} \mathrm{~N}$ имеет окисление, о чем свидетельствует асимметричная форма дублета In $3 d_{5}$. При этом степень окисления наноколонн $\operatorname{In}_{x} \mathrm{Ga}_{1-x} \mathrm{~N}$, выращенных на пористом кремнии (образец $\left.a \_p o r\right)$, немного выше, чем для образца, выращенного на кристаллическом кремнии.

\section{3. Результаты растровой электронной и атомно-силовой микроскопии (АСM)}

Микроскопия сколов гетероструктур, выполненная с помощью электронного микроскопа, позволила уточнить толщину слоя и оценить полученную наноколончатую структуру. Как видно из полученных данных (рис. 2), наноколончатый слой, выращенный на кристаллическом кремнии, имеет толщину $\sim 170 \mathrm{Hм}$, в то время как на пористом слое его толщина составила $\sim 140$ нм. Кроме
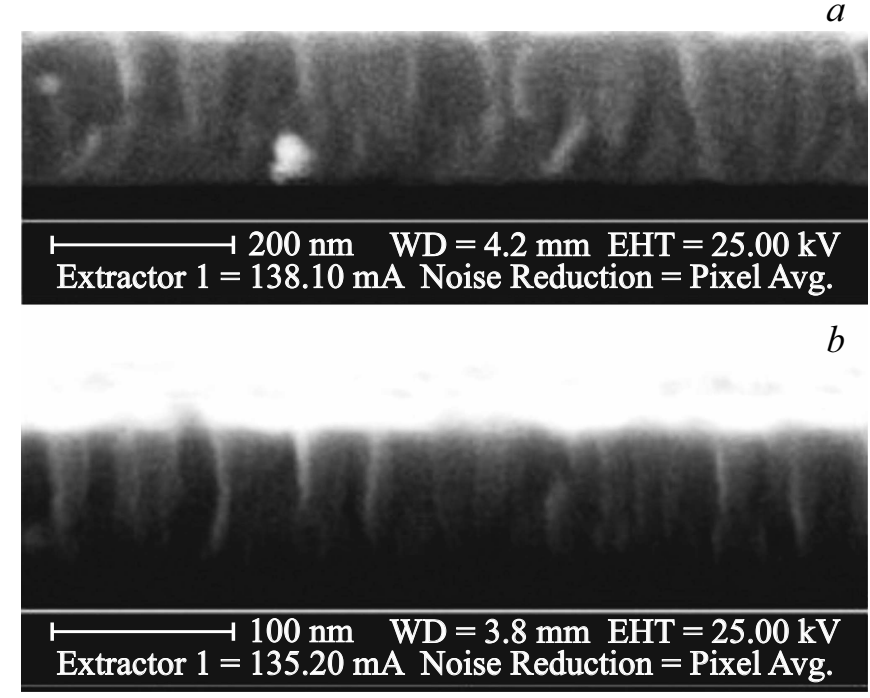

Рис. 2. Результаты исследований сколов гетероструктур методом электронной микроскопии: $a$ - образец $a \_c r y s t ;$ $b$ - образец $a \_$por.

того, хорошо видно, что при росте на пористом слое степень коалесценции наноколонн $\operatorname{In}_{x} \mathrm{Ga}_{1-x} \mathrm{~N}$ значительно выше.

Эти данные находятся в хорошем согласии с результатами атомно-силовой микроскопии, представленной на рис. 3. Эксперимент показывает, что плотность наноколонн $\mathrm{In}_{x} \mathrm{Ga}_{1-x} \mathrm{~N}$ на микроучастках поверхности значительно выше для образца, выращенного на слое пористого кремния. Кроме того, анализ формы распределения размеров наноколонн $\operatorname{In}_{x} \mathrm{Ga}_{1-x} \mathrm{~N}$ на поверхности двух типов образцов (рис. 3) показывает, что в случае эпитаксиального роста такой структуры на por-Si подавляющее количество наноколонн (> 75\%) имеют размер $\sim 40$ нм, в то время как при росте на $c$-Si размер наноколонн статистически варирьируется в пределах $20-60 \mathrm{HM}$.

\section{4. Дифрактометрические исследования}

Применение рентгеновской дифрактометрии позволяет надежно определять параметры кристаллической решетки эпитаксиальных слоев в гетероструктуре, а также их релаксацию [17-19].

На рис. 4 приведены обзорные дифрактограммы для исследованных образцов a_cryst и $a \_p o r$. Хорошо заметно, что на дифрактограммах обоих гетероструктур наиболее интенсивной линией является дифракция от плоскости (111) подложки Si. Что же касается остальных максимумов, то они являются дифракционными рефлексами (0002) и (0004) твердого раствора $\operatorname{In}_{x} \mathrm{Ga}_{1-x} \mathrm{~N}$ $(x \sim 0.33)$ с вюрцитной симметрией кристаллической решетки.

Следует отметить, что на малых углах дифракции у образцов наблюдается низкоинтенсивный галообразный 

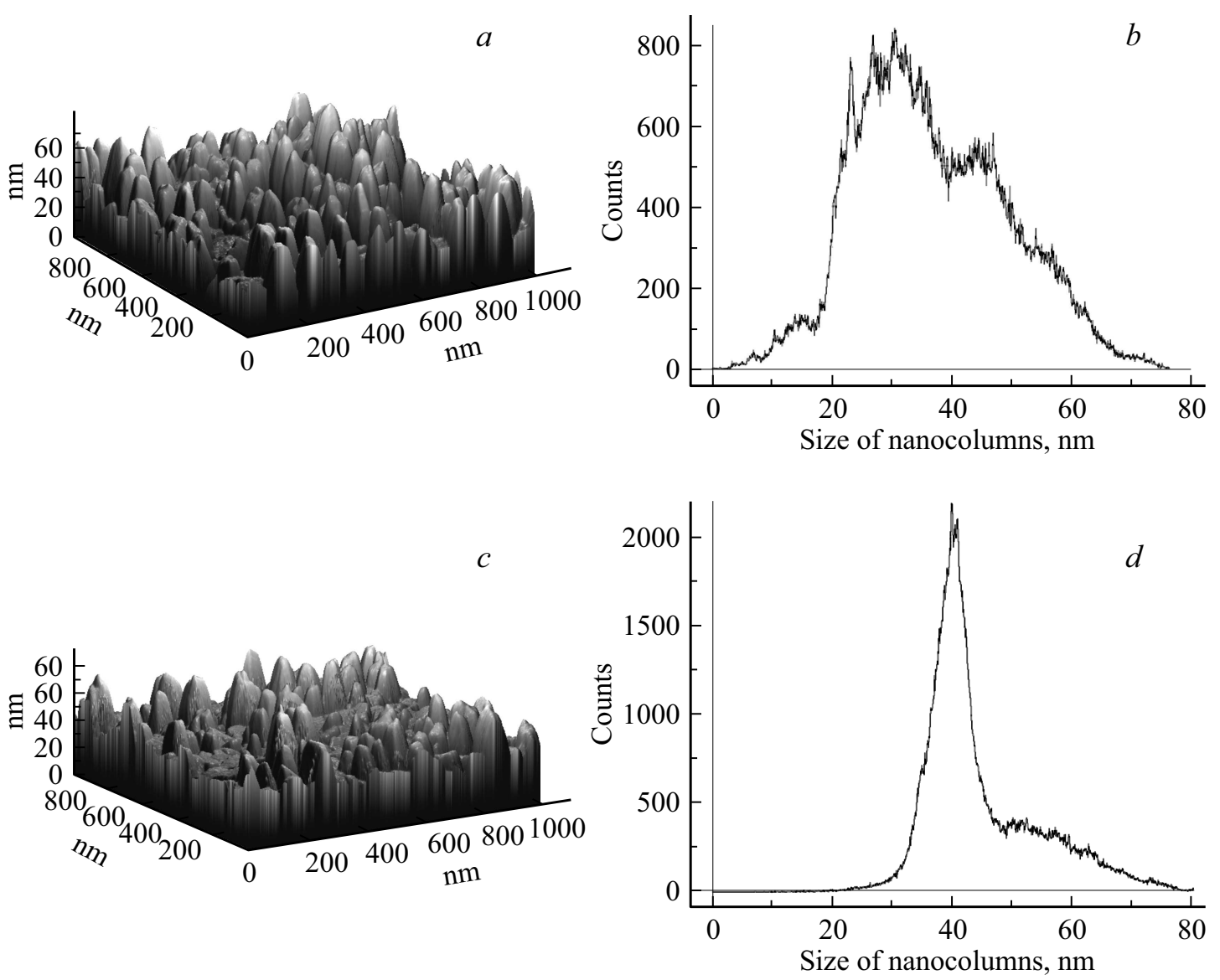

Рис. 3. Результаты атомно-силовой микроскопии. $a$ - рельеф поверхности образца $a \_c r y s t ; b-$ распределение размеров наноколонн $\operatorname{In}_{x} \mathrm{Ga}_{1-x} \mathrm{~N}$ на поверхности образца $a \_c r y s t ; c-$ рельеф поверхности образца $a \_p o r ; ~ d-$ распределение размеров наноколонн $\operatorname{In}_{x} \mathrm{Ga}_{1-x} \mathrm{~N}$ на поверхности образца $a \_p o r$.

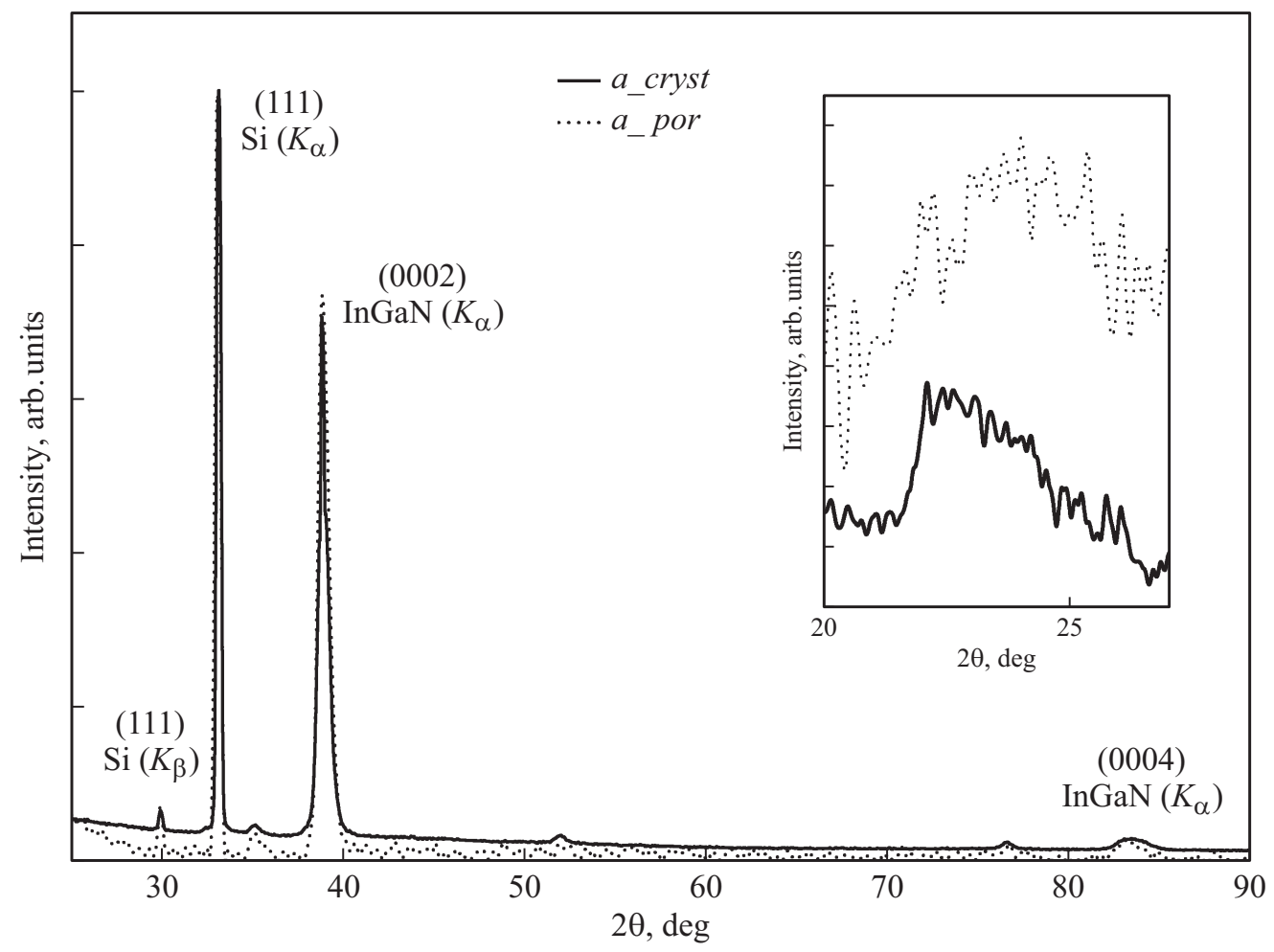

Рис. 4. Обзорная дифракция образцов гетероструктур. 

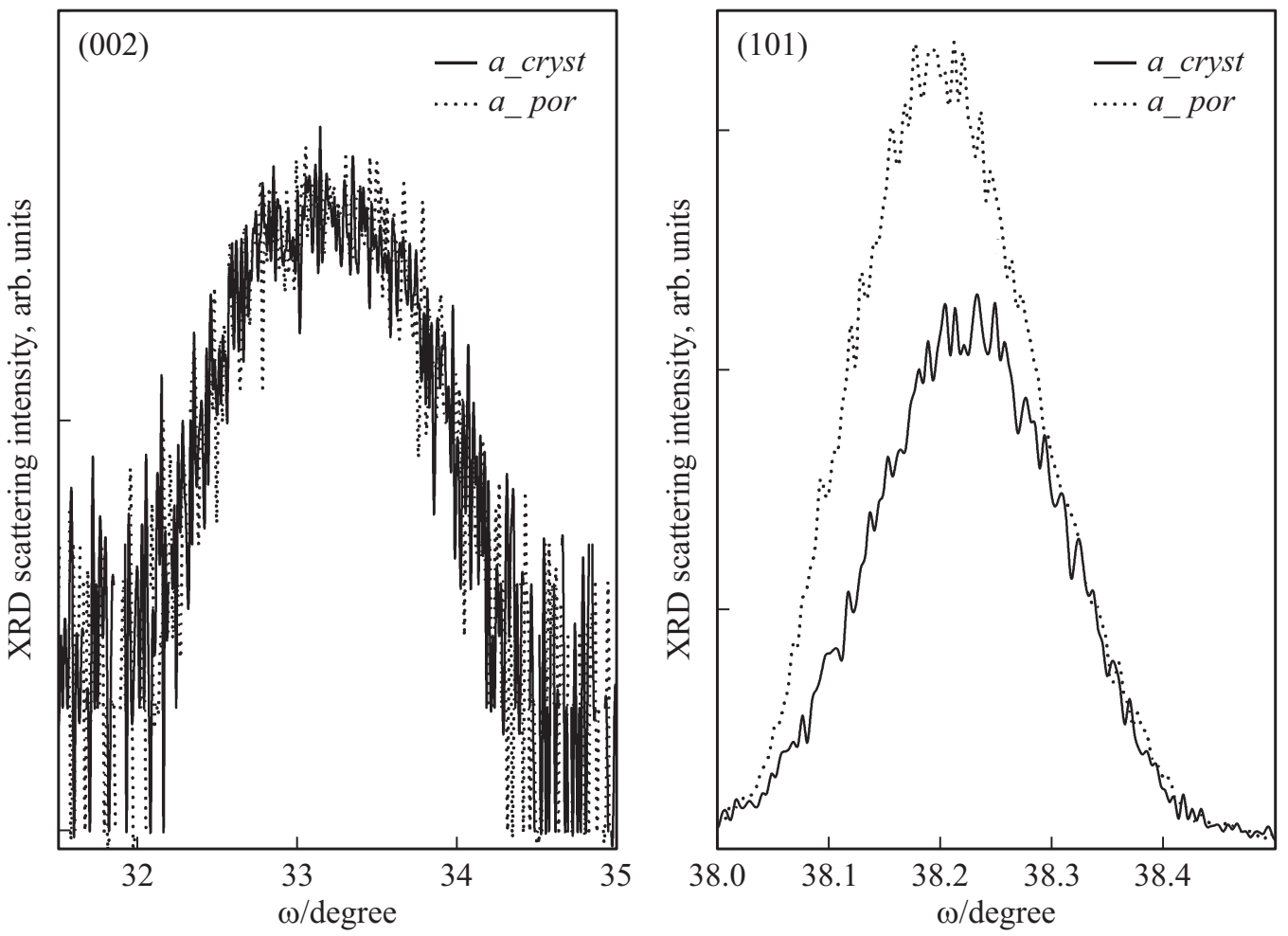

Рис. 5. Экспериментальные кривые качания для исследованных образцов в геометрии $2 \theta-\omega$. $a-$ скан для рефлекса (0002); $b$ - скан для рефлекса $(10 \overline{1} 1)$.

фон, что свидетельствует о существовании аморфной фазы в составе эпитаксиальной пленки (см. вставку к рис. 4). Учитывая, что интенсивность этой дифракции выше для образца $a \_p o r$, можно доподлинно утверждать, что объем аморфной фазы больше для гетероструктуры, выращенной на слое пористого кремния por-Si.

Хорошо известно, что основные параметры, характеризующие кристаллическую структуру наноколончатой пленки $\mathrm{In}_{x} \mathrm{Ga}_{1-x} \mathrm{~N}$, такие как параметры решетки, их несоответствие параметрам монокристаллической подложки и возникающие в этом случае напряжения, могут быть определены из прямых измерений кривых качания.

На рис. 5 представлены экспериментальные кривые качания для исследованных образцов в геометрии $2 \theta-\omega$.

Симметричный скан был выполнен для рефлекса

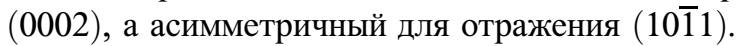

Используя уравнение Бреггов $2 d_{h k(k+l) l} \sin \theta=n \lambda$ из данных для симметричного рефлекса (0002) и соотношения $d_{000 l}=\frac{c}{l}$ можем найти параметр $c$.

Далее, используя рассчитанное значение параметра $c$, а также данные асимметричного отражения (101) и соотношение

$$
d_{h k-(k+l) l}=\frac{1}{\sqrt{\frac{4}{3 a^{2}}\left(h^{2}+k^{2}+h k\right)+\frac{l^{2}}{c^{2}}}},
$$

можем найти параметр $a$.
Рассчитанные параметры кристаллических решеток представлены в табл. 2.

Для напряженной вюрцитной гетероструктуры $\operatorname{In}_{x} \mathrm{Ga}_{1-x} \mathrm{~N}$ компоненты деформации $\varepsilon_{x x}$ и $\varepsilon_{z z}$ (параллельный и перпендикулярный к поверхности пленки) определяются как

$$
\varepsilon_{x x}=\frac{a-a_{0}(x)}{a_{0}(x)}, \quad \varepsilon_{z z}=\frac{c-c_{0}(x)}{c_{0}(x)},
$$

где $a$ и $c$ - измеренные параметры решетки, тогда как $a_{0}(x)$ и $c_{0}(x)$ - полностью релаксированные параметры решетки.

Зависимость для параметров решеток твердого раствора $\mathrm{In}_{x} \mathrm{Ga}_{1-x} \mathrm{~N}$, а также для коэффициента Пуассона может быть записана в виде линейного закона Вегарда по методике, аналогичной той, которая была многократно использована в наших предыдущих работах [20,21]:

$$
\begin{aligned}
& c_{0}(x)=c_{0}^{\mathrm{GaN}}+\left(c_{0}^{\mathrm{InN}}-c_{0}^{\mathrm{GaN}}\right) x, \\
& a_{0}(x)=a_{0}^{\mathrm{GaN}}+\left(a_{0}^{\mathrm{InN}}-a_{0}^{\mathrm{GaN}}\right) x, \\
& v=0.210+(0.399-0.210) x,
\end{aligned}
$$

где $c_{0}^{\mathrm{GaN}}=5.1850 \AA, a_{0}^{\mathrm{GaN}}=3.1892 \AA, c_{0}^{\mathrm{InN}}=5.7233 \AA$, $a_{0}^{\mathrm{InN}}=3.5378 \AA, v^{\mathrm{GaN}}=0.210, v^{\mathrm{InN}}=0.399$. Здесь параметры решеток и коэффициенты Пуассона бинарных соединений, рассчитанные из констант тензора упругих напряжений $C_{i j}$, приведены на основе данных [22] и 
Таблица 2. Результаты рентгеноструктурного анализа

\begin{tabular}{|c|c|c|c|c|c|c|c|}
\hline & \multicolumn{2}{|c|}{ Параметры } & \multirow{2}{*}{$\varepsilon_{x x}$} & \multirow{2}{*}{$\mathcal{E}_{z z}$} & \multirow{2}{*}{$\begin{array}{c}\rho_{\text {screw }} \\
\mathrm{cm}^{-1}\end{array}$} & \multirow{2}{*}{$\begin{array}{l}\rho_{\text {edge }} \\
\mathrm{cm}^{-1}\end{array}$} & \multirow{2}{*}{ Искажение } \\
\hline & $c$ & $a$ & & & & & \\
\hline $\begin{array}{c}a \_c r y s t \\
a \_p o r\end{array}$ & $\begin{array}{l}5.4005 \\
5.4156\end{array}$ & $\begin{array}{l}3.0178 \\
3.0253\end{array}$ & $\begin{array}{l}-0.087 \\
-0.086\end{array}$ & $\begin{array}{l}7.06 \cdot 10^{-3} \\
7.04 \cdot 10^{-3}\end{array}$ & $\begin{array}{l}3.15 \cdot 10^{10} \\
3.10 \cdot 10^{10}\end{array}$ & $\begin{array}{l}1.97 \cdot 10^{9} \\
1.60 \cdot 10^{9}\end{array}$ & $\begin{array}{l}-0.081 \\
-0.082\end{array}$ \\
\hline
\end{tabular}

практически совпадают с наиболее часто используемыми значениями [23,24].

Основываясь на теории упругости, деформация в плоскости роста $\varepsilon_{x x}$ связана линейно с деформацией в направлении роста $\varepsilon_{z z}$ через соотношение:

$$
D=\frac{\varepsilon_{z z}}{\varepsilon_{x x}}
$$

где $D$ - коэффициент дисторсии (искажения) [25].

Рассчитанные нами с учетом (3)-(5) значения компонент деформации $\varepsilon_{x x}$ и $\varepsilon_{z z}$, а также величина коэффициента дисторсии, возникающей в наноколончатом слое, приведены в табл. 2.

Эпитаксиальный рост $\operatorname{In}_{x} \mathrm{Ga}_{1-x} \mathrm{~N}$ в виде наноколончатой структуры, а также разница в параметрах решетки подложки и твердого раствора и коэффициентах их теплового расширения во время роста приводят к появлению большого числа дефектов в виде краевых и винтовых дислокаций. Эти дислокации проходят вдоль оси $c$ кристаллической решетки, а их векторы Бюргерса характеризуют искажения, вызванные в кристаллической решетке дислокациями [26]. Как уже было сказано ранее, дислокации играют важную роль в релаксации напряжений кристаллических решеток при создании финальных оптоэлектронных устройств.

Ввиду того что появление различных по типу дислокаций вызывает уширение дифракционных рефлексов, для того чтобы оценить плотность дислокаций в $\operatorname{In}_{x} \mathrm{Ga}_{1-x} \mathrm{~N}$, зачастую используют данные рентгеновских кривых качания.

Для расчета плотности краевых дислокаций используют асимметричный скан, в то время как для оценки плотности винтовых дислокаций применяют симметричное отражение [26].

В соответствии с данными работ $[27,28]$ плотность дислокаций может быть вычислена исходя из следующего соотношения:

$$
\begin{gathered}
\rho_{\text {Dis }}=\rho_{\text {edge }}+\rho_{\text {screw }}, \\
\rho=\frac{\beta_{(h k l)}^{2}}{4.35 b^{2}}, \\
b_{\text {screw }}^{2}=2.65 \cdot a_{0}^{2}(x), \\
b_{\text {edge }}^{2}=1.3 a_{0}^{2}(x) .
\end{gathered}
$$

Здесь $a_{0}^{2}(x)$ - ненапряженный параметр твердого раствора $\operatorname{In}_{x} \mathrm{Ga}_{1-x} \mathrm{~N}$ с составом $x$.
Величины векторов Бюргерса $b_{\text {screw }}$ и $b_{\text {edge }}$ для $\mathrm{In}_{x} \mathrm{Ga}_{1-x} \mathrm{~N}$ вычислены на основе предложенных в [28] подходов и линейного закона Вегарда для параметров решетки (4).

Рассчитанная плотность краевых и винтовых дислокаций для исследованных в работе образцов представлена в табл. 2.

Хорошо известно, что для $\mathrm{In}_{x} \mathrm{Ga}_{1-x} \mathrm{~N}$ типичная плотность винтовых дислокаций, характеризующихся вектором Бюргерса $\langle 001\rangle$, имеет величину $10^{8}-10^{10} \mathrm{~cm}^{-2}$, а краевых (вектор Бюргерса 1/3〈110 $) \sim 10^{8}-10^{9} \mathrm{~cm}^{-2}$. Сравнивая полученные нами результаты и уже известные литературные данные можно отметить, что плотность дислокаций в слое $\operatorname{In}_{x} \mathrm{Ga}_{1-x} \mathrm{~N}$ с наноколончатой морфологией лежит на границе значений, характерных для эпитаксиального сплошного слоя $\mathrm{In}_{x} \mathrm{Ga}_{1-x} \mathrm{~N}$.

\section{5. Полюсные фигуры}

Для того чтобы определить симметрию и кристаллографическую ориентацию наноколонок $\operatorname{In}_{x} \mathrm{Ga}_{1-x} \mathrm{~N}$ выращенных на подложке $c$-Si и подложке с пористым слоем por-Si, мы провели измерение полюсных фигур для (111) рефлекса $\mathrm{Si},(0002)$ и (1012) рефлексов $\operatorname{In}_{x} \mathrm{Ga}_{1-x} \mathrm{~N}$. Экспериментальные данные представлены на рис. 6. Как видно из полученных результатов для обоих образцов распределения интенсивности рентгеновского луча на полюсной фигуре около отражения (111) от Si и (0002) отражения от InGaN расположены в центрах полюсных фигур и никаких других рефлексов не наблюдается. Это означает, что ось с наностолбиков $\operatorname{In}_{x} \mathrm{Ga}_{1-x} \mathrm{~N}$ перпендикулярна плоскостям $\mathrm{Si}(111)$.

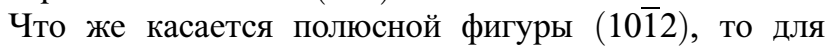
обоих образцов характерно присутствие на ней трех внутренних рефлексов (при меньших Chi), которые соответствуют отражению от (220) плоскости $\mathrm{Si}$, a также шести внешних более интенсивных отражений от (1012) плоскости $\operatorname{In}_{x} \mathrm{Ga}_{1-x} \mathrm{~N}$. Одновременное присутствие рефлексов $\operatorname{In}_{x} \mathrm{Ga}_{1-x} \mathrm{~N}$ и $\mathrm{Si}$ обусловлено близостью межплоскостных расстояний. Исходя из распределения интенсивностей рентгеновского отражения на полюсной фигуре (10, 2 ) следует, что в реальном пространстве направления $\langle\overline{1} 10\rangle$ в кремнии параллельны $\langle 100\rangle$ направлениям в $\operatorname{In}_{x} \mathrm{Ga}_{1-x} \mathrm{~N}$. Другими словами, для обоих образцов подложка $\mathrm{Si}$ задает ориентацию растущей наноколончатой структуры.

Обратим внимание на то, что интенсивность рефлексов (220) Si по отношению к интенсивности отражений 

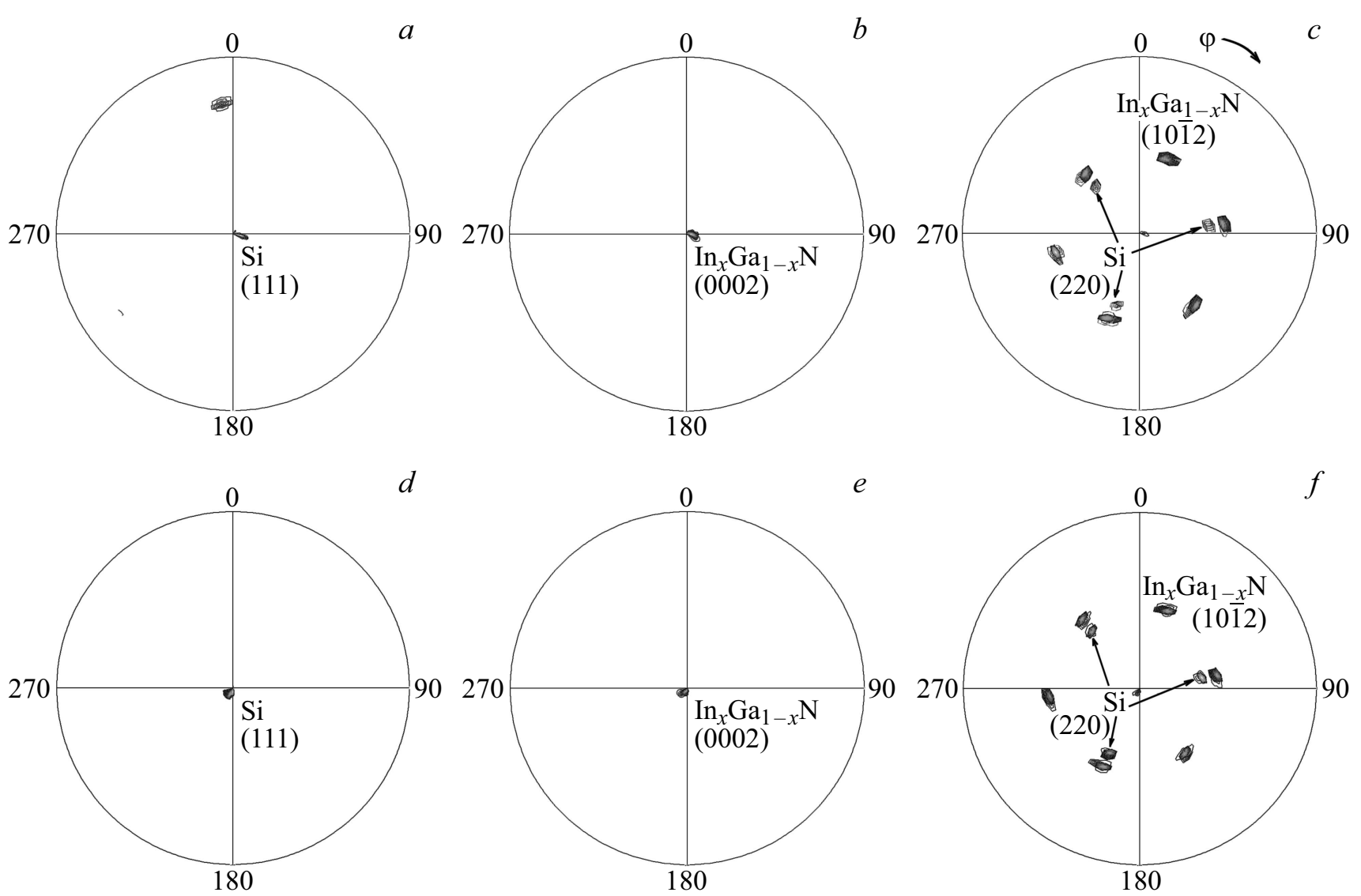

Рис. 6. Экспериментальные полюсные фигуры: $(a, d)-(111)$ рефлекс $\mathrm{Si} ;(b, e)-(0002)$ рефлекс $\operatorname{In}_{x} \mathrm{Ga}_{1-x} \mathrm{~N}$; $(c, f)-(10 \overline{1} 2)$ рефлекс $\operatorname{In}_{x} \mathrm{Ga}_{1-x} \mathrm{~N} .(a, b, c)$ - образец $a \_$cryst; $(d, e, f)$ - образец $a \_p o r$.

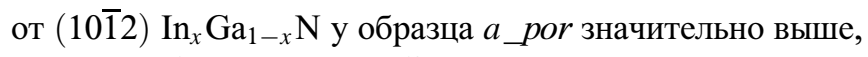
чем для образца $a \_$cryst. Это позволяет предположить, что направление преимущественной ориентации роста наноколонок $\operatorname{In}_{x} \mathrm{Ga}_{1-x} \mathrm{~N}$ ближе к направлению ориентации подложки у гетероструктуры, выращенной на пористом слое por-Si (образец $a_{\text {_ }}$ por).

Следует также отметить, что ширина (1012) брэгговских отражений для $\operatorname{In}_{x} \mathrm{Ga}_{1-x} \mathrm{~N}$ значительно больше, чем у брэгговских отражений (220) от подложки $\mathrm{Si}$. Это означает, что кристаллографическая однородность наноколонок $\operatorname{In}_{x} \mathrm{Ga}_{1-x} \mathrm{~N}$ хуже по отношению к кристаллографической однородности подложки $\mathrm{Si}$, что в общем-то и не вызывает сомнений. При этом полуширина брэгговских отражений для слоя $\operatorname{In}_{x} \mathrm{Ga}_{1-x} \mathrm{~N}$ выращенного на por- $\mathrm{Si}$, меньше, чем полуширина аналогичного рефлекса для слоя $\operatorname{In}_{x} \mathrm{Ga}_{1-x} \mathrm{~N}$, выращенного на $c$ - $\mathrm{Si}$.

\section{4. Заключение}

С использованием метода молекулярно-пучковой эпитаксии с плазменной активацией азота на подложке $\mathrm{Si}(111)$ и por-Si(111) нами были выращены интегрированные гетероструктуры с наноколончатой морфологией пленки $\operatorname{In}_{x} \mathrm{Ga}_{1-x} \mathrm{~N}$. C привлечением комплекса структурных и микроскопических методов анализа (высокоразре- шающая рентгеновская дифракция, сканирующая электронная и атомно-силовая микроскопия) были получены следующие результаты.

Полученная наноколончатая пленка $\operatorname{In}_{x} \mathrm{Ga}_{1-x} \mathrm{~N}$ имеет вюрцитную симметрию кристаллической решетки и состав $x \sim 0.33$. Состав наноколонок подтвержден тремя независимыми методами: рентгеновского микроанализа, фотоэлектронной и фотолюминесцентной спектроскопии. Эпитаксиальный слой $\operatorname{In}_{x} \mathrm{Ga}_{1-x} \mathrm{~N}$ имеет $p$-тип проводимости, при этом величина холловской концентрации примеси находится на уровне $(3.0-3.64) \cdot 10^{19} \mathrm{~cm}^{-3}$, а подвижность носителей заряда $\sim 104 \mathrm{~cm}^{2} / \mathrm{B} \cdot \mathrm{c}$. Однако при одинаковом уровне подвижности носителей заряда для обоих образцов концентрация носителей заряда на $\sim 20 \%$ выше для пленки, выращенной на пористом кремнии.

Результаты АСМ показали, что плотность наноколонн $\mathrm{In}_{x} \mathrm{Ga}_{1-x} \mathrm{~N}$ на микроучастках поверхности значительно выше для образца $a \_p o r$, выращенного на слое пористого кремния. При этом рост наноколончатой структуры на слое пористого кремния приводит к тому, что средний размер более чем на $75 \%$ наноколонн составляет $\sim 40$ нм, в то время как при росте на подложке кристаллического кремния размер наноколонн на поверхности статистически варирьируется в пределах 20-60 нм. 
Расчет, выполненный из данных рентгеновских кривых качания, показал, что рост наноколонн $\operatorname{In}_{x} \mathrm{Ga}_{1-x} \mathrm{~N}$ на пористом слое приводит к снижению величины компонент деформации $\varepsilon_{x x}$ и $\varepsilon_{z z}$, а также плотности краевых и винтовых дислокаций по сравнению с величинами аналогичных коэффициентов для слоя $\operatorname{In}_{x} \mathrm{Ga}_{1-x} \mathrm{~N}$ на монокристаллическом Si. Снижение плотности краевых дислокаций для образца на пористом слое отмечено на уровне $25 \%$ по сравнению с гетероструктурой на кристаллическом кремнии $\mathrm{Si}(111)$.

Результаты исследования рентгеновских полюсных фигур показали, что для обоих образцов подложка $\mathrm{Si}$ задает ориентацию растущей наноколончатой структуры. Однако преимущественная ориентация роста наноколонок $\operatorname{In}_{x} \mathrm{Ga}_{1-x} \mathrm{~N}$ ближе к направлению ориентации подложки у гетероструктуры, выращенной на пористом слое $\mathrm{Si}$. Кристаллографическая однородность наноколонок $\mathrm{In}_{x} \mathrm{Ga}_{1-x} \mathrm{~N}$, выращенных на пористом слое, лучше, чем для $\operatorname{In}_{x} \mathrm{Ga}_{1-x} \mathrm{~N}$ на монокристаллической подложке $\mathrm{Si}$.

Таким образом, на основании комплекса полученных данных можно сделать вывод, что описанный нами подход к интеграции $\mathrm{A}^{\mathrm{III}} \mathrm{N}$ соединений и кремниевой подложки имеет высокий потенциал и достаточно эффективно может быть внедрен в стандартные технологии оптоэлектроники.

Работа выполнена при поддержке гранта президента РФ МД-188.2017.2. Ростовые эксперименты проводились в рамках выполнения государственного задания Министерства образования и науки Российской Федерации № 16.9789.2017/БЧ. Работа в части исследований управления морфологией и составом монолитной и пористой подложек выполнена при финансовой поддержке ФТИ им. А.Ф. Иоффе В части диагностики интегрированных структур работа поддержана грантом № 11.4718.2017/8.9 Министерства образования и науки России в рамках государственного задания вузам в сфере научной деятельности на 2017-2019 гг.

We acknowledge the Karlsruhe Nano Micro Facility (KNMF, www.kit.edu/knmf) of the Forschungszentrum Karlsruhe for provision of access to instruments at their laboratories.

\section{Список литературы}

[1] C. Li, Z. Ji, J. Li, M. Xu, H. Xiao, X. Xu. Sci. Rep., 7, 15301 (2017).

[2] S. Albert, A. Bengoechea-Encabo, P. Lefebvre, M.A. SanchezGarcia, E. Calleja, U. Jahn, A. Trampert. Appl. Phys. Lett., 99, 131108 (2011).

[3] S. Keating, M.G. Urquhart, D.V.P. McLaughlin, J.M. Pearce. Cryst. Growth. Des., 11, 565 (2011).

[4] A. Kikuchi, M. Tada, K. Miwa, K. Kishino. Proc. Integrated Optoelectronic Devices (San Jose, California, United States, 2006) v. 6129, p. 612905.

[5] W. Zhang, X. Zhang, Y. Wang, F. Hu. Opt. Mater., 72, 422 (2017).
[6] T. Kano, J. Yoshida, R. Miyagawa, Y. Mizuno, T. Oto, K. Kishino. Electron. Lett., 51, 2125 (2015).

[7] K. Vanhollebeke, I. Moerman, P. Van Daele, P. Demeester. Prog. Cryst. Growth. Charact. Matters, 41, 1 (2000).

[8] S. Shetty, S.M. Shivaprasad. Proc. IEEE 2nd International Conference on Emerging Electronics (ICEE) (Bangalore, India, 2014) p. 1.

[9] C. Hahn, Z. Zhang, A. Fu, C.H. Wu, Y.J. Hwang, D.J. Gargas, P. Yang. ACS Nano, 5, 3970 (2011).

[10] P.V. Seredin, A.S. Lenshin, D.S. Zolotukhin, I.N. Arsentyev, A.V. Zhabotinskiy, D.N. Nikolaev. Phys. E. Low-Dim. Syst. Nanostructures, 97, 218 (2018).

[11] P.V. Seredin, A.S. Lenshin, D.S. Zolotukhin, I.N. Arsentyev, D.N. Nikolaev, A.V. Zhabotinskiy. Phys. B: Condens. Matter, 530, 30 (2018).

[12] P.V. Seredin, A.S. Lenshin, V.M. Kashkarov, A.N. Lukin, I.N. Arsentiev, A.D. Bondarev, I.S. Tarasov. Mater. Sci. Semicond. Proc., 39, 551 (2015).

[13] A.S. Lenshin, P.V. Seredin, B.L. Agapov, D.A. Minakov, V.M. Kashkarov. Matter. Sci. Semicond. Proc., 30, 25 (2015).

[14] A.S. Len'shin, V.M. Kashkarov, P.V. Seredin, B.L. Agapov, D.A. Minakov, V.N. Tsipenyuk, E.P. Domashevskaya. Techn. Phys., 59, 224 (2014).

[15] V.M. Kashkarov, A.S. Len'shin, P.V. Seredin, B.L. Agapov, V.N. Tsipenuk. J. Surf. Investig. $X$-ray Synchrotron Neutron Techn., 6, 776 (2012).

[16] Z.L. Fang, Q.F. Li, X.Y. Shen, H. Xiong, J.F. Cai, J.Y. Kang, W.Z. Shen. J. Appl. Phys., 115, 043514 (2014).

[17] P.V. Seredin, A.V. Glotov, E.P. Domashevskaya, A.S. Lenshin, M.S. Smirnov, I.N. Arsentyev, D.A. Vinokurov, A.L. Stankevich, I.S. Tarasov. Semiconductors, 46, 719 (2012).

[18] P.V. Seredin, V.E. Ternovaya, A.V. Glotov, A.S. Len'shin, I.N. Arsent'ev, D.A. Vinokurov, I.S. Tarasov, H. Leiste, T. Prutskij. Phys. Solid State, 55, 2161 (2013).

[19] P.V. Seredin, E.P. Domashevskaya, I.N. Arsentyev, D.A. Vinokurov, A.L. Stankevich, T. Prutskij. Semiconductors, 47, 1 (2013).

[20] P.V. Seredin, A.S. Lenshin, A.V. Glotov, I.N. Arsentyev, D.A. Vinokurov, I.S. Tarasov, T. Prutskij, H. Leiste, M. Rinke. Semiconductors, 48, 1094 (2014).

[21] P.V. Seredin, E.P. Domashevskaya, I.N. Arsentyev, D.A. Vinokurov, A.L. Stankevich. Semiconductors, 47, 7 (2013).

[22] S. Adachi. Properties of semiconductor alloys: group-IV, III$V$ and II-VI semiconductors, ed. by P. Capper, S. Kasap, A. Willoughby (U.K., Wiley, 2009).

[23] M.A. Moram, Z.H. Barber, C.J. Humphreys. J. Appl. Phys., 102, 023505 (2007)

[24] A.F. Wright. J. Appl. Phys., 82, 2833 (1997).

[25] P.V. Seredin, A.V. Glotov, E.P. Domashevskaya, I.N. Arsentyev, D.A. Vinokurov, A.L. Stankevich, I.S. Tarasov. Semiconductors, 44, 1106 (2010).

[26] I. Booker, L. Rahimzadeh Khoshroo, J.F. Woitok, V. Kaganer, C. Mauder, H. Behmenburg, J. Gruis, M. Heuken, H. Kalisch, R.H. Jansen. Phys. Status Solidi C, 7, 1787 (2010).

[27] T. Metzger, R. Höpler, E. Born, O. Ambacher, M. Stutzmann, R. Stömmer, M. Schuster, H. Göbel, S. Christiansen, M. Albrecht, H.P. Strunk. Philos. Mag. A, 77, 1013 (1998).

[28] S.K. Hong, T. Yao, B.J. Kim, S.Y. Yoon, T.I. Kim. Appl. Phys. Lett., 77, 82 (2000).

Редактор Г.А. Оганесян 
The effect of por-Si buffer layer on the

structure and morphology of

$\ln _{x} \mathrm{Ga}_{1-x} \mathrm{~N} / \mathrm{Si}(111)$ epitaxial

heterostructures

P.V. Seredin ${ }^{1}$, D.L. Goloshchapov' ${ }^{1}$, D.S. Zolotukhin ${ }^{1}$,

M.A. Kondrashin ${ }^{1}$, A.S. Lenshin ${ }^{1}$, Yu.Yu. Khudyakov' ${ }^{1}$, A.M. Miserov ${ }^{2}$, I.N. Arsentyev ${ }^{3}$, A.N. Beltiukov ${ }^{4}$,

Harald Leiste ${ }^{5}$, Monica Rinke ${ }^{5}$

${ }^{1}$ Voronezh State University, 394006 Voronezh, Russia

${ }^{2}$ St. Petersburg National Research Academic University of the Russian Academy of Sciences,

194021 St. Petersburg, Russia

${ }^{3}$ loffe Institute,

194021 St. Petersburg, Russia

${ }^{4}$ Physico-Technical Institute of the Ural Branch

of the Russian Academy of Science,

426000 Izhevsk, Russia

${ }^{5}$ Karlsruhe Nano Micro Facility,

76344 Eggenstein-Leopoldshafen, Germany

Abstract We demonstrate successfully growth of the $\operatorname{In}_{x} \mathrm{Ga}_{1-x} \mathrm{~N}$ nanorods layer on traditional and compliant, with preformed nanoporous $\mathrm{Si}$ layer, $c$-Si(111) substrates by plasma-assisted molecular beam epitaxy. Usage of the structural and microscopic analysis methods revealed that $\operatorname{In}_{x} \mathrm{Ga}_{1-x} \mathrm{~N}$ nanocolumns grown on porous $\mathrm{Si}$ layer have some advantages in comparison with ones, grown on traditional $c$-Si substrate. Por-Si layer better defines preferential $\operatorname{In}_{x} \mathrm{Ga}_{1-x} \mathrm{~N}$ nanocolumns growth orientation closer to $\mathrm{Si}(111)$ substrate orientation. Moreover, por-Si layer helps to achieve nanocolumns with higher crystalline homogeneity and more unified lateral diameters distribution $(\sim 40 \mathrm{~nm})$ in comparison with ones, grown on traditional $c$-Si substrate. Usage of the compliant substrate with por-Si layer leads to a decrease in the value of the $\varepsilon_{x x}$ and $\varepsilon_{z z}$ strain components, and helps to suppress edge and screw threading dislocation generation through the growth which results in better crystalline quality of $\operatorname{In}_{x} \mathrm{Ga}_{1-x} \mathrm{~N} /$ por- $\mathrm{Si}(111)$ layer in comparison with $\operatorname{In}_{x} \mathrm{Ga}_{1-x} \mathrm{~N} / c-\operatorname{Si}(111)$ ones. $\operatorname{In}_{x} \mathrm{Ga}_{1-x} \mathrm{~N} /$ por-Si(111) layer showed $20 \%$ higher carrier concentration and $25 \%$ more intensive photoluminescence spectra than $\operatorname{In}_{x} \mathrm{Ga}_{1-x} \mathrm{~N} / c-\mathrm{Si}(111)$ layer. 